\title{
Electron Lithography In Glasses by STEM
}

\author{
Nan Jiang, Gary Hembree and John C. H. Spence \\ Department of Physics and Astronomy, \\ Arizona State University, Tempe AZ 85287-1504
}

It has been known for almost three decades that the field emission STEM may be used for direct-write inorganic lithography with sub-nanometer resolution in a variety of materials [1]. For example, the direct conversion of silica to silicon [2] and the formation of a zoneplate lens using patterned holes in $\mathrm{MgF}_{2}$ [3] have been reported. In the past few years, we have extended this technique to the formation of patterned nano-structures in glasses [4]. Electron lithography should be more efficient in glasses than in crystals, because there remain unfilled sites in three-dimensional space in glasses, which may accommodate atoms, and the relaxation associated with excitation processes can more easily yield configurational changes. Therefore, the mechanisms involved in the processes in glasses and crystals are different. Recently, we have used this technique to generate patterned nano-rings of dopants in several silicate glasses [5]. In the following, we present an example of nano-ring formation in an $\mathrm{Nb}_{2} \mathrm{O}_{5}-\mathrm{Li}_{2} \mathrm{O}-\mathrm{SiO}_{2}$ glass using the Vacuum Generators "Midas" HB501S UHV field emission STEM [6] at Arizona State University.

Figure 1 shows the ADF images of Niobium ring structures formed by the electron probe in an $\mathrm{Nb}_{2} \mathrm{O}_{5}-\mathrm{Li}_{2} \mathrm{O}-\mathrm{SiO}_{2}$ glass. The diameter and the width of the rings are about 10 and 2 $\mathrm{nm}$, respectively. Figure 2 is the backscattered secondary electron image (BSEI) of the same area. The ADF intensity profiles across one ring parallel and perpendicular to the edge of the sample is given in Fig. 3. Since the electron transparent samples were obtained by crushing a bulk glass, and picking up small pieces suspended in acetone using a holy carbon film across a copper grid, a wedge-shaped intensity profile is expected. Sputtering facilities have been constructed for the formation of glass films of constant thickness on silicon nitride membranes. Figure 4 shows EELS spectra recorded inside and on the ring using an electron probe diameter less than $0.5 \mathrm{~nm}$. It is seen that they are significantly different. On the ring, $\mathrm{Nb}$ has very high intensity, but inside the ring, there is little $\mathrm{Nb}$ left. The exact stoichiometry of any oxide in the ring has yet to be determined. Normally, the thickness of rings should be much smaller than the diameter. In our case, however, it is dependent on the thickness of the sample. In other words, selecting the thickness of the sample can control the aspect ratio of the ring.

The formation of rings in glasses by electron irradiation is due to an ionization and fieldinduced atom migration process. In glasses, some oxygens, called non-bridging oxygens, are partially covalently bound to only one $\mathrm{Si}$, and ionically bound to metal ions. Electron excitation can easily break the ionic bond between the non-bridging oxygen and the metal ion. As a result, the metal ion is released from the network, and diffuses away from the irradiated area driven by repulsive electrostatic forces. We are exploring the possibility of using these rings to make materials with negative refractive index. The optical absorption spectrum of these rings has been computed in collaboration with F.J. Garcia de Abajo [5]. This work is supported by NSF DMR9814055. 


\section{References}

[1] A. N. Broers et al. Electron Microscopy 1978, Vol III, edited by J. M. Sturgess, Microscopical Society of Canada, p.343.

[2] G. Chen, C. Boothroyd and C. Humphreys, Appl. Phys. Lett. 62 (1003) 1949

[3] Y. Itoh, et al. Nature 394 (1998) 49.

[4] N. Jiang, et al., Appl. Phys. Lett. 77 (2000) 3956; and 80 (2002) 2005.

[5] N. Jiang, et al., submitted to Appl. Phys. Lett. (2003).

[6] G, Hembree, C.Koch and J. C. H. Spence (2003) Micros and Microan. In press.

\section{$20 \mathrm{~nm}$}

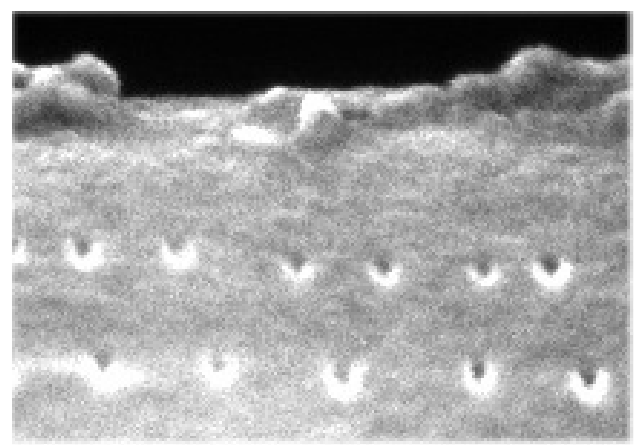

Figure 1 ADF image showing nano-rings.
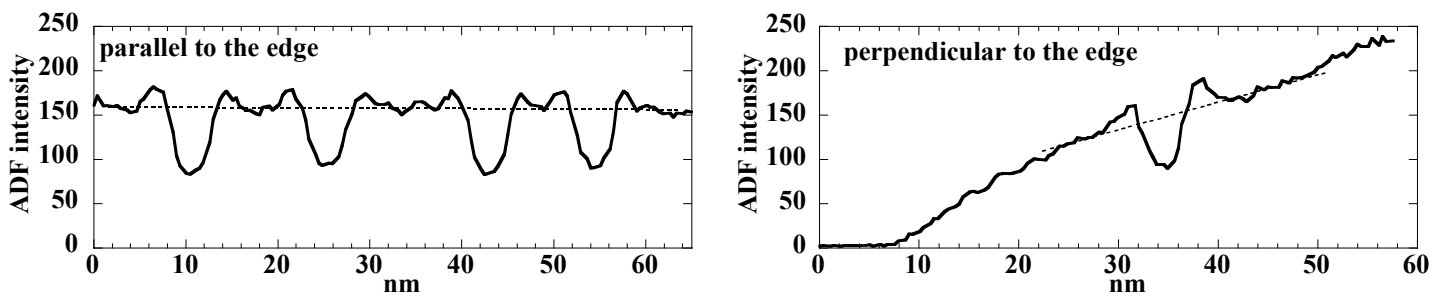

Figure 3 ADF intensity profiles across rings, parallel (left) and perpendicular (right) to the edge of the sample. Intensity levels before forming rings are indicated by dotted lines.

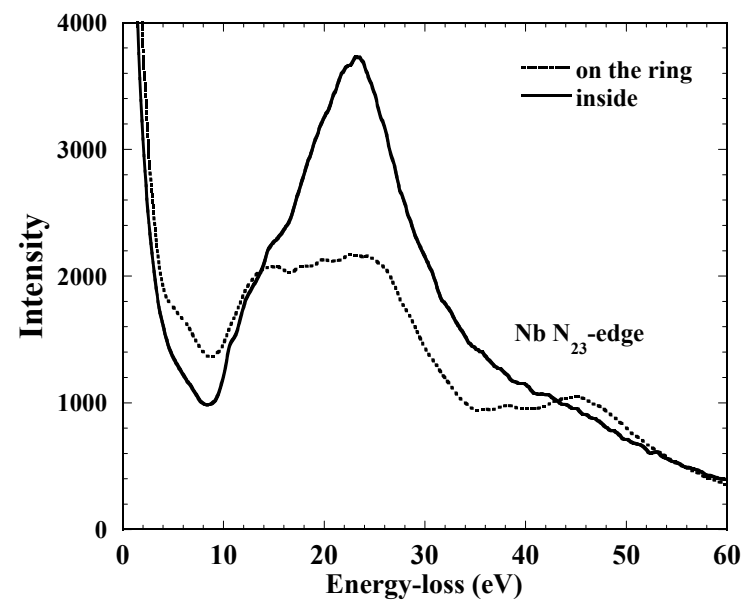

Figure 4 Comparison of EELS spectra on (upper curve) and inside (lower curve) a nanoring. 